\title{
The Influence of Humidity on the Resistance Structures with Graphene Sensor Layer
}

\author{
T. Pustelny ${ }^{a, *}$, M. Setkiewicz ${ }^{a}$, S. Drewniak ${ }^{a}$, E. Maciak $^{a}$, A. StolarczyK $^{b}$,

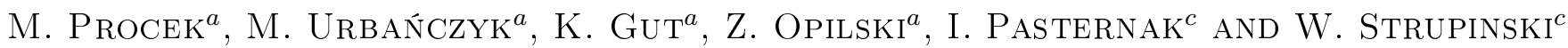 \\ ${ }^{a}$ Department of Optoelectronics, Silesian University of Technology, Akademicka 2, 44-100 Gliwice, Poland \\ ${ }^{b}$ Department of Physical Chemistry and Technology of Polymers, Silesian University of Technology \\ Strzody 9, 44-100 Gliwice, Poland \\ ${ }^{c}$ Institute of Electronic Materials Technology (ITME), Wólcz'yńska 133, 01-919 Warsaw, Poland
}

In the paper the results of investigations are presented concerning the influence of humidity of air on the resistance of a gas sensor structure with a graphene layer. The affects of nitrogen dioxide and humidity action on graphene were studied. We indicated that humidity might play an important role in determining the gas sensing properties of the graphene layer. In the paper it has been shown that in the case of a nitrogen oxide sensor, the reaction of $\mathrm{NO}_{2}$ with water vapour can generate permanent defects in graphene.

PACS: 07.07.Df, 42.81.Pa, 82.47.Rs

\section{Introduction}

In recent years many works have been published concerning the applicability of graphene in gas sensors [1-8]. Most developments were focused on the sensitivity, selectivity, and stability of graphene sensors applied in artificial conditions. New technologies of graphene considerably enhanced the sensitivity and stability of gas sensors, but contributed relatively little towards a solution of the cross sensitivity. Any sensor can experience cross sensitivity. This can be used to advantage wishing to detect groups of compounds or using cross sensitivity for calibration. It is important to know the proper way of application so that a correct solution can be adopted. Fluctuations of the content of steam in atmospheric air and the unknown ability to adsorb it by a sensor layer are the most interfering factor in the actual operation of devices for detecting gases in the atmosphere.

Spin-polarized density functional theory (DFT) analyses have shown that water molecules adsorbed on graphene cause defects in it which facilitate to electrons tunneling of the bandgap and cause widening a graphene bandgap to $\approx 2 \mathrm{eV}$ [1]. In Ref. [2] the authors exposed the graphene film to humidity and created successfully a bandgap in graphene. The bandgap increases with the increase of humidity and saturates at $\approx 0.206 \mathrm{eV}$ at the humidity level of $c a .0 .312 \mathrm{~kg} \mathrm{~kg}^{-1}$, consistent with the theoretical result [1]. Therefore, the graphene swells and shrinks in relation to the relative humidity level. It is

\footnotetext{
* corresponding author; e-mail: Tadeusz.Pustelny@polsl.pl
}

believed that this swelling and shrinking is the cause of resistance changes observed at various humidity levels $[3,4]$. As a result, the changes in resistances are directly enhanced by the amount of water vapour absorbed by the graphene $[5,6]$.

This paper presents preliminary results of investigations of the sensitivity of the resistance structures with a graphene layer on nitrogen dioxide $\mathrm{NO}_{2}$ action in synthetic air with the presence of water vapour.

\section{Technology applied for the production of graphene}

The graphene deposited on resistance interdigital electrode structures was obtained at ITME in Warsaw. The graphene was arranged on the surface of a copper foil, $23 \mu \mathrm{m}$ thick, in the course of chemical sedimentation from the gaseous phase (CVD method). This process was carried out in two stages [9, 10]. First, in order to improve the quality of the substrate and to augment the size of the copper grains, the substrate was soaked in an Ar atmosphere at a temperature of about $1000^{\circ} \mathrm{C}$. Then the substrate was heated up for several minutes at $1020^{\circ} \mathrm{C}$ with a simultaneous flow of $\mathrm{H}_{2}$ and $\mathrm{C}_{3} \mathrm{H}_{3}$ as the source of carbon. Finally the copper foil, covered with graphene, was kept in the switched-off furnace for several hours to cool down to room temperature. In order to separate the graphene from the copper foil and to transfer it onto the interdigital electrode structure, on the surface of the copper sample (covered with graphene), by means of the spin-coating method a thin layer of poly(methyl methacrylate) (PMMA) was 
deposited. PMMA prevents graphene from cracking and damages which might arise in the course of etching the copper foil [9]. For the purpose of etching the copper structure $\mathrm{PMMA} /$ graphene/Cu was immersed in a solution of ammonia persulphate. After the copper foil had been thoroughly etched, the polymer with graphene was additionally cleaned in order to remove the remaining metal and insoluble organic impurities [10]. Next the graphene layer was transferred from PMMA to the interdigital resistance transducers. In order to ensure a thorough contact of graphene with the substrate, the structure was heated for several minutes at a temperature of $100^{\circ} \mathrm{C}$ in air and then kept for several hours in vacuum. Finally the PMMA layer was carefully removed by solving it in acetone. The parameters of the process of transferring graphene were chosen in such a way that a formation of gaps and cracks between the graphene layer and the surface of the interdigital electrode structure would be prevented.

\section{Resistance structure with graphene}

Graphene (obtained in the process described above) was deposited on a resistance interdigital transducer. Figure 1 presents the resistance transducer with graphene but modified by means of a $3 \mathrm{~nm}$ layer of palladium.

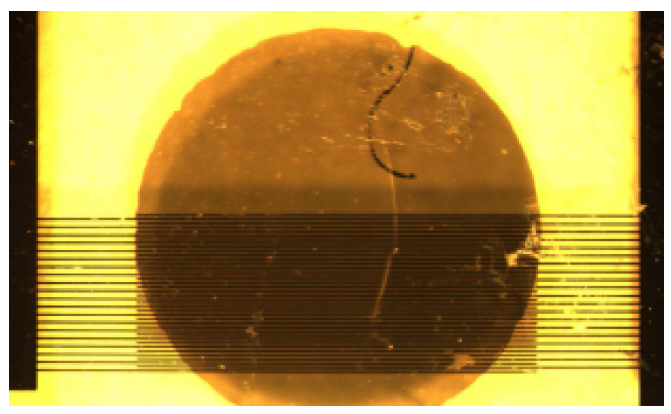

Fig. 1. Interdigital resistance transducer with a layer of graphene modified by $3 \mathrm{~nm}$ palladium layer.

For measurements of the resistance of a structure with a graphene layer a high-quality measurement system of the type AGILENT 34970 was applied. The total resistance of the sensor structure with a graphene layer amounted at ambient temperature (about $20^{\circ} \mathrm{C}$ ) to about $10 \Omega$. The measurements were carried out with an accuracy of $\pm 0.5 \times 10^{-3} \Omega$. It is to be stressed that the values of electrical resistance obtained in all these measurements concern the total resistance of the structure (the resistance of the grahene layer and of the interdigital transducer).

\section{Investigations of the sensitivity of graphene exposed to water vapour}

Graphene is a hydrophilic material. Water adsorbed on its surface causes in result that such a structure behaves as a semiconductor in which the width of the bandgap can depend on the amount of adsorbed water. Data quoted in literature [1] show that such a semiconductor structure formation occurs immediately upon its contact with moisture. Figure 2 presents the sensitivity of a structure with graphene affected by $\mathrm{NO}_{2}$. The first reaction of the sensor structure with graphene on the action of moist air is presented in Fig. 2. (It is an initial part of the characteristics in Fig. 2, between 1000-1500 s, before the first contact of this structure with $\mathrm{NO}_{2}$.)

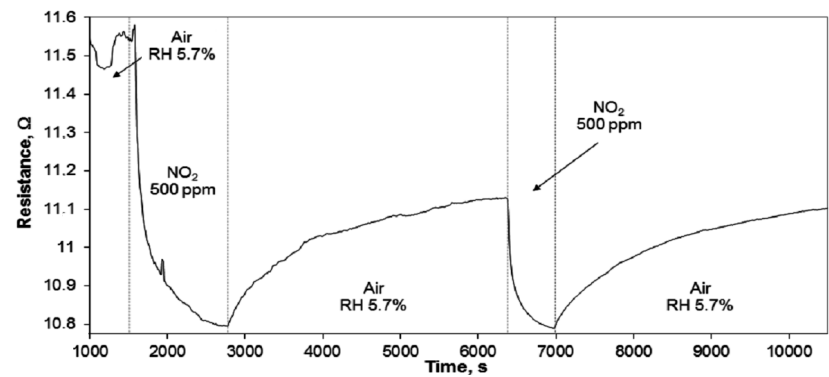

Fig. 2. Sensitivity of the sensor structure with graphene to the presence of $\mathrm{NO}_{2}$ in the atmosphere of synthetic air (temperature of the structure $50^{\circ} \mathrm{C}$, humidity $\mathrm{RH}$ about $5 \%$ ).

The resistance is maintained constant when only air flows through the channel and decreases when $\mathrm{NO}_{2}$ flows through it. When the molecules of $\mathrm{NO}_{2}$ gases are absorbed onto graphene, as bringing electrons from graphene, holes are created in graphene. It leads to a decrease of the resistance [3].

It ought to be additionally stressed that the effect of increased conductivity is constant and that it does not fade entirely after the exchange of gas. This phenomenon may be accounted for by the fact that the adsorption of $\mathrm{NO}_{2}$ into the graphene structure is constant or, which seems to be even more probable, that some part of $\mathrm{NO}_{2}$ reacts with the adsorbed water forming nitric acid. Nitric acid oxidizes graphene and generates stable defects in the graphene structure. Consequently after every subsequent experimental exchange of gases we obtained similar values of electrical conductivity of the sensor structure.

Figure 3 illustrates the sensitivity of the sensor structure modified by means of a $3 \mathrm{~nm}$ thickness layer of palladium to the presence of $\mathrm{NO}_{2}$ in the atmosphere of synthetic air. The character of the behaviour of the resistance structure with graphene (Fig. 2) and the structure with a deposited $3 \mathrm{~nm}$ layer of palladium is more or less the same. The graphene structure modified with palladium seems to react to $\mathrm{NO}_{2}$ faster than the not modified graphene structure.

\section{Sensitivity of graphene to water vapour in the atmosphere of synthetic air}

The resistance of the structures decreased at switching from humid air to dry air. However the response of the 


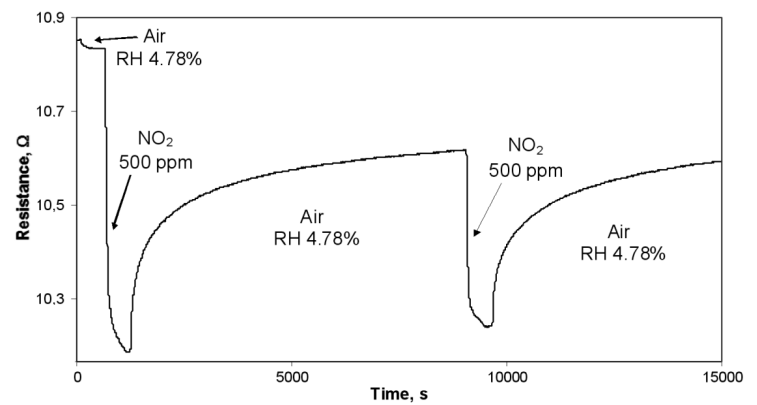

Fig. 3. Sensitivity of the sensor structure with graphene modified by $3 \mathrm{~nm}$ PD layer to the presence of $\mathrm{NO}_{2}$ in the atmosphere of synthetic air (temperature of the structure $50{ }^{\circ} \mathrm{C}$, humidity $\mathrm{RH}$ about $5 \%$ ).

sensing structure was faster and higher in the case of the graphene $/ \mathrm{Pd}$ variant. The results of investigations are presented in Figs. 4, 5 and 6.

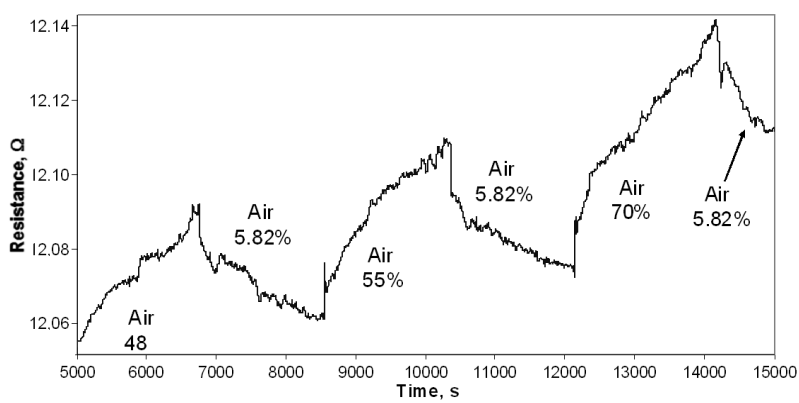

Fig. 4. Sensitivity of the sensor structure with graphene to the presence of steam in the atmosphere of synthetic air (temperature of the structure $120^{\circ} \mathrm{C}$, humidity $\mathrm{RH}$ about $6-70 \%$ ).

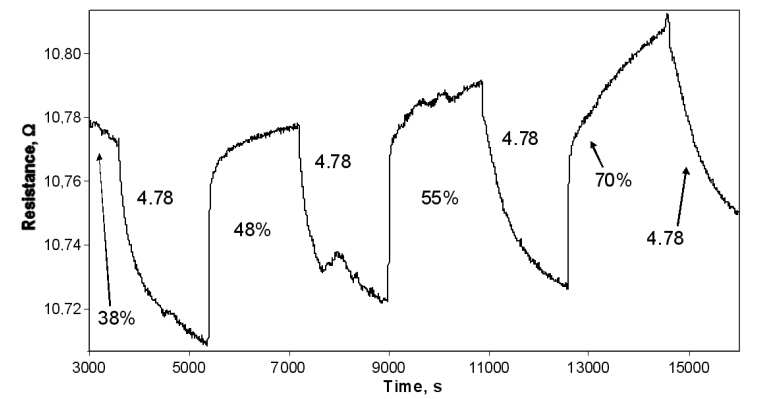

Fig. 5. Sensitivity of the sensor structure with graphene modified by a $3 \mathrm{~nm} \mathrm{Pd}$ layer to the presence of steam in the atmosphere of synthetic air (temperature of the structure $50{ }^{\circ} \mathrm{C}$, humidity $\mathrm{RH}$ about $6-70 \%$ ).

Investigations have proved explicitly that the presence of steam in the atmosphere of synthetic air obviously influences the resistance of the structure with a sensor layer of graphene. Measurements indicated that in comparison with the effect of nitrogen dioxide the influence of steam

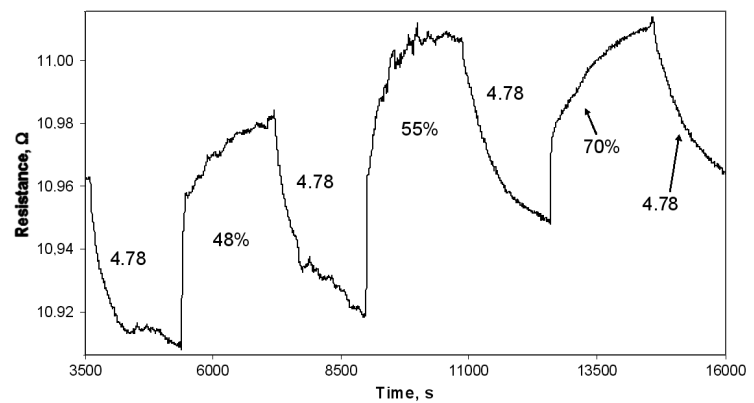

Fig. 6. Sensitivity of the sensor structure with graphene modified by a $3 \mathrm{~nm} \mathrm{Pd}$ layer to the presence of steam in the atmosphere of synthetic air (temperature of the structure $120^{\circ} \mathrm{C}$, humidity $\mathrm{RH}$ about $6-70 \%$ ).

is also of essential importance. The problem of the affect of steam on the non-spectral gas sensors has been already dealt with by other researchers [11-18]. The performed investigations indicate distinctly that the problem of eliminating steam from the tested gaseous medium is also very important in the application of graphene (or modified graphene) in resistance gas sensors.

\section{Conclusions}

We presented a novel nanocomposite chemosensitive material consisting of chemically derived graphene and graphene modified by thin (with $3 \mathrm{~nm}$ thickness) palladium nanoparticles. In the presence of moisture, graphene behaves as a semiconductor and as such a semiconductor structure ought to be considered the presented sensor in the moist air (i.e. in future conditions of its work). Nitrogen dioxide reacting with water adsorbed on graphene forms nitric acid capable to undergo reaction of oxidation of graphene generating permanent defects in graphene layer of sensing structures. The problem of reducing uncontrolled presence of humidity in investigated gaseous atmosphere in resistance gas sensors based on graphene (and its derivatives) must be necessarily resolved.

\section{Acknowledgments}

The work was partially sponsored by the National Centre for Scientific Researches and Development NCBiR within the grant OR00017912.

\section{References}

[1] J. Berashevich, T. Chakraborty, Phys. Rev. B 80, 3404 (2009).

[2] F. Yavari, C. Kritzinger, C. Gaire, L. Song, H. Gulapalli, T. Borca-Tasciuc, P.M. Ajayan, N. Koratkar, Small 6, 2535 (2010).

[3] F. Schedin, A.K. Geim, S.V. Morozov, E.E. Hill, P. Blake, M.I. Katsnelson, K.S. Novoselov, Nature Mater. 6, 652 (2007). 
[4] R.S. Sundaram, C. Gomez-Navarro, K. Balasubramanian, M. Burghard, K. Kern, Adv. Mater. 20, 3050 (2008).

[5] J. Cassidy, S. Pons, J. Janata, Anal. Chem. 58, 1757 (1986).

[6] Byung Hwan Chu, C.F. Lo, J. Nicolasi, C.Y. Chang, V. Chen, W. Strupinski, S.J. Pearton, F. Ren, Sensor Actuators B 157, 500 (2011).

[7] P.E. Gaskell, H.S. Skulason, W. Strupinski, T. Szkopek, Opt. Lett. 35, 3336 (2010).

[8] E. Hill, A. Vijayaragahvan, K. Novoselov, IEEE Sensors J. 11, 3161 (2011)

[9] A. Reina, H. Son, L. Jiao, B. Fan, M.S. Dresselhaus, Z. Liu, J. Kong, J. Phys. Chem. C 112, 17741 (2008).

[10] X. Liang, B.A. Sperling, I. Calizo, G. Cheng, C.A. Hacker, Q. Zhang, Y. Obeng, K. Yan, H. Peng, Q. Li, X. Zhu, H. Yuan, A.R.H. Walker, Z. Liu, Lian-Mao Peng, C.A. Richter, ACS Nano 5, 9144 (2011).

[11] E. Maciak, Z. Opilski, T. Pustelny, M. Bednorz, J. Phys. IV, (France) 129, 131 (2005).
[12] T. Pustelny, B. Pustelny, Opto-Electron. Rev. 10, 193 (2002).

[13] Z. Opilski, T. Pustelny, E. Maciak, M. Bednorz, A. Stolarczyk, M. Jadamiec, Bull. Pol. Acad. Sci. Techn. Sci. 53, 151 (2005).

[14] W. Jakubik, M. Urbanczyk, E. Maciak, T. Pustelny, Bull. Pol. Acad. Sci. Techn. Sci. 56, 133 (2008).

[15] M. Urbanczyk, E. Maciak, K. Gut, T. Pustelny, W. Jakubik, Bull. Pol. Acad. Sci. Techn. Sci. 59, 401 (2011)

[16] K. Barczak, T. Pustelny, Z. Zycki, T. Blazejczak, Acta Phys. Pol. A 116, 250 (2009).

[17] K. Gut, J. Phys. IV, (France) 129, 109 (2005).

[18] Z. Bielecki, J. Janucki, A. Kawalec, J. Mikolajczyk, N. Palka, M. Pasternak, T. Pustelny, T. Stacewicz, J. Wojtas, Metrology Measur. Systems 19, 3 (2012). 